\title{
Impacto del agua asociada a la producción de una explotación petrolera sobre la comunidad fitoperifítica del rio Acacias (Meta, Colombia) durante la temporada de Iluvias
}

\section{Impact of wastewater discharge from an oil company on the phytoperiphyton community of Acacias river (Meta, Colombia) during raining season}

\author{
Néstor F. Vera-Parra ${ }^{1}$; Lili J. Marciales-Caro², Angélica M. Otero-Paternina ${ }^{3}$, \\ Pablo E. Cruz-Casallas ${ }^{4 *}$, Yohana M. Velasco-Santamaría ${ }^{5 *}$ \\ 'Biologo, Fundación Natura, Bogotá, Colombia \\ 2Biologa, Universidad de los Llanos, \\ ${ }^{3}$ Profesional en Acuicultura, cMSc, Universidad de los Llanos, \\ ${ }^{4}$ Médico Veterinario Zootecnista, MSc, PhD, Universidad de los Llanos, \\ ${ }^{5}$ Médico Veterinaria, MRes, cPhD, Instituto de Biología, Universidad del Sur de Dinamarca, Odense, \\ Campusvej 55, DK-5000, Dinamarca. \\ "Grupo de Investigación sobre Reproducción y Toxicología de Organismos Acuáticos - GRITOX, \\ Instituto de Acuicultura, Universidad de los Llanos \\ *E-mail: ymvelascos@gmail.com
}

Recibido: Junio 23 de 2010. Aprobado: Marzo 2 de 2011

\begin{abstract}
RESUMEN
Con el fin de determinar los efectos del agua asociada a la producción de la industria petrolera, vertida al río Acacias en el Departamento del Meta - Colombia, sobre la abundancia y riqueza de la comunidad fitoperifítica, así como sus efectos sobre las variables físicas y químicas del agua del afluente, se realizaron muestreos de las comunidades asociadas a sustratos rocosos durante la época de lluvias (Julio 27 Agosto 10 de 2009), seleccionando tres puntos principales: ca. $200 \mathrm{~m}$ antes del vertimiento, en el sitio de vertimiento y sobre el lecho del río después de la zona de mezcla del vertimiento (ca. $200 \mathrm{~m}$ ), evaluándose los parámetros físicos y químicos y el componente abiótico. Se encontraron diferencias altamente significativas $(P<0.001)$ para los factores bióticos como riqueza y abundancia en el lugar del vertimiento, así como en las variables físicas y químicas del mismo sitio, lo cual permite inferir la existencia de eventuales efectos deletéreos de estos vertimientos sobre el ecosistema acuático, favoreciendo el afloramiento de algas Cyanophycecae en el lugar del vertimiento.
\end{abstract}

Palabras clave: bioindicador, explotación petrolera, fitoperifiton, vertimientos, algas. 


\section{ABSTRACT}

The aim of this study was to determine the effects of water associated with the oil industry production, which is discharged into the Acacias River, Meta - Colombia. For this purpose, the abundance and richness of the phytoperyphyton community and the effects on physical and chemical water variables of the tributary receiving the wastewater discharges were evaluated throughout an evaluation of the algae communities associated with rocky substrates during the rainy season (July 27 to August 10, 2009). Three main points were selected: ca. $200 \mathrm{~m}$ before the wastewater discharge, the wastewater discharges site and, the river after the mixing of wastewater discharge (ca. $200 \mathrm{~m}$ after the mixing). The physical and chemical parameters and the abiotic component were evaluated in each site. Highly significant differences $(P<0.001)$ for biotic factors, such as richness of algae and abundance, and also physical and chemical variables in the wastewater discharges site were observed. These results could infer the possible deleterious effects of these wastewater discharges on the aquatic ecosystem allowing the higher abundance of Cyanophycecae in this site.

Key words: bioindicador, oil industry, phytoperiphyton, wastewater discharge, algae.

\section{INTRODUCCIÓN}

En los ecosistemas acuáticos en general, la presencia de agentes externos o contaminantes de tipo orgánico o inorgánico provocan modificaciones en las características físicas y químicas del agua, lo cual puede afectar directamente la composición y distribución de las comunidades acuáticas (Roldán, 1992). Los organismos acuáticos sometidos a una sustancia tóxica responden de manera individual; sin embargo, generan respuestas a nivel poblacional, comunitario y sobre el ecosistema. La magnitud de estos cambios dependen principalmente de la duración de la perturbación, de su intensidad, de su naturaleza y de las condiciones iníciales del ecosistema acuático (Pinilla, 2000). En los últimos años se han venido implementando métodos para evaluar la calidad del agua, basados en la utilización de comunidades biológicas (Barbour et al., 1999). En este contexto, un bioindicador acuático es considerado como aquel organismos cuya presencia y abundancia señala algún proceso o estado del sistema en el cual habita (Pinilla, 2000).

Las algas responden de manera rápida a los impactos antropogénicos como el exceso de nutrientes y sustancias tóxicas, convirtiéndose en buenos indicadores de la calidad del agua. Uno de los principales indicadores biológicos en ecosistemas acuáticos es el perifiton, el cual está compuesto por una comunidad de microbiota autotrófica (algas), heterotrófica (bacterias, hongos,

32 protozoos y animales) y detritos orgánicos e inorgánicos adheridos a un sustrato vivo o muerto (Lowe, 1996; Wetzel, 2001). Dentro del perifiton, la fracción conformada por las algas es el principal componente autótrofo del sistema y es denominada fitoperifiton. Esta comunidad está constituida principalmente por algas verdes (Chlorophyceae), diatomeas (Bacillariophyceae), algas verde-azules (Cyanophyceae) y muy ocasionalmente por algas rojas (Rhodophyceae) (Lowe, 1996). La composición de esta comunidad está relacionada tanto directa como indirectamente con variables ambientales tales como luminosidad, turbulencia, disponibilidad y tipo de nutrientes, características físicas y químicas del agua y las variaciones en las fluctuaciones en el nivel del agua tanto en escalas espaciales como temporales (Lowe, 1996). Adicionalmente es una importante fuente de materia orgánica autóctona, por su capacidad para fijar carbono para las comunidades heterotróficas (Wetzel, 2001).

Dentro de las cadenas tróficas, el fitoperifiton es una de las bases alimenticias de algunas especies de peces de importancia económica como algunas especies de loricáridos, así como de insectos y macroinvertebrados, importantes en la dinámica de los ecosistemas acuáticos (Roldan, 1992).

Una potencial fuente de contaminación ambiental lo constituyen las compañías petroleras, las cuales 
en sus procesos industriales inyectan agua a presión para obtener el petróleo. Aunque el agua utilizada es sometida a procesos de descontaminación no hay estudios publicados sobre los efectos de los vertimientos petroleros provenientes de esta industria sobre las comunidades fitoplanctónicas de las áreas circundantes; sin embargo, existen varias evidencias que los derivados de hidrocarburos poseen efectos adversos sobre el medio ambiente

\section{MATERIALES Y MÉTODOS}

\section{Área de Estudio}

Las muestras de fitoperifiton fueron colectadas de sustratos rocosos del rio Acacias, localizado en el sector de Chichimene, Municipio de Acacias (Meta, Colombia), localizado a una altura de $498 \mathrm{msnm}$ y temperatura promedio anual de $25^{\circ} \mathrm{C}$. Este afluente recibe el vertimiento del agua asociada a la producción de la estación petrolera "Chichimene". El trabajo de campo se realizó durante la temporada de lluvias (Julio 27 - Agosto 10 de 2009), efectuándose 5 muestreos a intervalos de 72 horas. Se seleccionaron tres puntos de muestreo: antes del vertimiento (ca. $200 \mathrm{~m}$ ), en el sitio de vertimiento ( $03^{\circ} 57^{\prime} 23.2^{\prime \prime} \mathrm{N}$ y $73^{\circ} 40^{\prime} 18.15^{\prime \prime}$ WO) y sobre el lecho del río después de la zona de mezcla del vertimiento (ca. 200 metros aguas abajo).

\section{Variables Biológicas}

Con relación al componente biótico se tomaron muestras cualitativas y cuantitativas de sustratos rocosos, por medio de remoción mecánica en un área de $8.8 \mathrm{~cm}^{2}$, utilizando para este fin una cuadrícula. Se realizaron 5 réplicas en cada caso, siendo cada roca una réplica. Las muestras cuantitativas fueron fijadas con solución de Grahm ( $0.1 \mathrm{~mL} / \mathrm{L})$ y las cualitativas con solución Transseau en proporción 1:1. Se realizó la caracterización preliminar de la comunidad por medio de observaciones con microscopio óptico (40x) (Nikon®

\section{RESULTADOS}

\section{Variables Biológicas}

Se encontró un total de 16 taxa distribuidas en 5 grupos algales, siendo el grupo Bacillariophyceae
(Oliveira-Filho et al., 2008; French-McCay, 2002). En consecuencia, el objetivo del presente estudio fue evaluar los efectos del agua asociada a la producción de un campo petrolero, vertida al rio Acacias (Meta - Colombia), sobre el ecosistema acuático, mediante la valoración de la comunidad fitoperifitica asociada a sustratos rocosos, durante la temporada de lluvias, y la incidencia de estos vertimientos sobre variables físicas y químicas del agua de afluente.

Eclipse E200) y posteriormente la cuantificación de los organismos por medio de la cámara de Sedgwick Rafter de 1.25 mL (Wetzel et al., 2001).

\section{Variables Físicas y Químicas}

Las variables físicas y químicas fueron medidas in situ en cada punto de muestreo, siendo las variables evaluadas: temperatura $\left({ }^{\circ} \mathrm{C}\right)$, conductividad $(\mu \mathrm{S} / \mathrm{cm})$, oxígeno disuelto $(\mathrm{mg} / \mathrm{L})$, sólidos totales disueltos $(\mathrm{g} / \mathrm{L})$ y $\mathrm{pH}$, las cuales fueron determinadas con una sonda multiparamétrica YSI 556 Professional Plus.

\section{Análisis Estadístico}

Se realizó un análisis de varianza de la riqueza y de las variables abióticas de cada uno de uno los sitios de muestreo, seguido de una prueba de Tukey tomando el nivel de significancia limite como 0.05 . Los efectos de las variables ambientales en las abundancias de las especies fitoperifíticas y su variación espacial, se analizaron con el método CCA (Análisis de Correspondencia Canónica) usando el programa CANOCO (Ter Braak y Smilaurer, 1998). Los análisis se basaron en los datos de la abundancia de especies fitoperifiticas y de concentraciones de las variables ambientales correspondientes. La significancia estadística de los ejes se estimó por el test de Monte Carlo con 9999 permutaciones.

el mayor contribuyente a la riqueza en los tramos del río antes y después del vertimiento, aportando 
entre el 30 y $60 \%$ de la riqueza en cada tramo de muestreo. El grupo Zygnematophyceae sólo hizo presencia en el tramo antes del vertimiento representando un valor muy bajo en la riqueza de este punto de muestreo (alrededor del $10 \%$ de la riqueza fitoperifitica). Por otra parte, se observó un afloramiento de algas Cyanophyceae en el lugar del vertimiento, alcanzando el $40 \%$ de la riqueza fitoperifitica en este lugar (Figura 1).

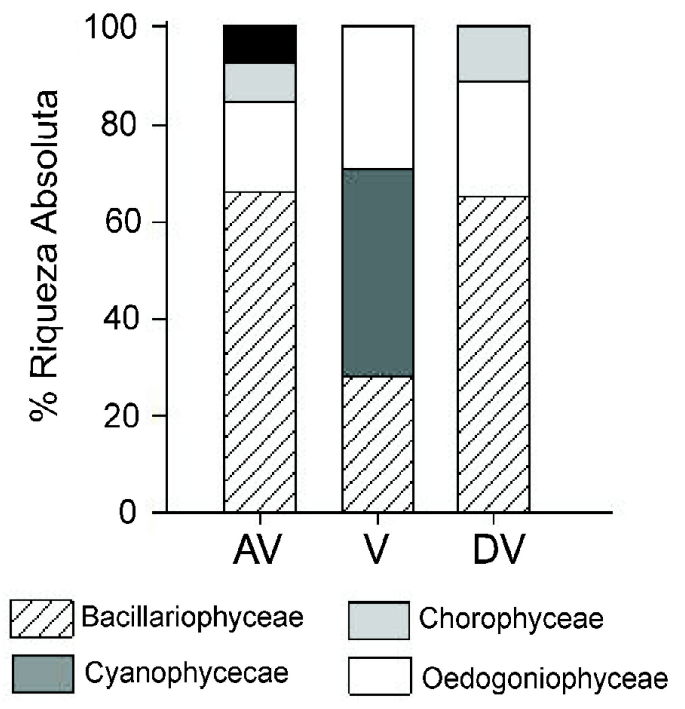

Zygnemathophyceae

Figura 1. Comparación de la riqueza de algas y su aporte en la riqueza de los grupos algales en cada uno de los puntos de muestreo del río Acacias durante época de lluvias. Antes del vertimiento (AV), en el vertimiento (V) y después del vertimiento (DV)

Algas del grupo Chorophyceae conservaron el porcentaje de su aporte a la riqueza en los tramos antes y después del vertimiento, siendo en los dos lugares alrededor del $10 \%$. Sin embargo, la presencia de este grupo en el lugar del vertimiento se ve reemplazada por organismos pertenecientes al grupo de las Cyanophyceae. La cantidad de individuos del género Oedogonium se mantuvo constante en cada punto de muestreo, por tal razón se tomó como un grupo aparte denominado Oedogoniophyceae y su aporte a la riqueza de cada lugar estuvo entre el 20 y el $30 \%$. La riqueza presentó diferencias significativas $(P<0.001)$ entre los tres lugares de muestreo, siendo la más alta en el lugar antes del vertimiento y la más baja abundancia en el sito del vertimiento. El resumen de las variables físicas y químicas y la riqueza fitoperifitica se presentan en la Tabla 1 y Tabla 2, respectivamente.

La abundancia relativa no varió de manera significativa en cada uno de los grupos algales en los tres lugares evaluados durante los cinco muestreos realizados (Figura 2). 
Tabla 1. Variables físicas, químicas y biológicas medidas en los sitios de muestreo del río Acacias durante época de lluvias. Se muestran las probabilidades del análisis de varianza. Valores expresados como media \pm desviación estándar

\begin{tabular}{|c|c|c|c|c|c|c|}
\hline & $\begin{array}{c}\text { Temperatura } \\
\left({ }^{\circ} \mathrm{C}\right)\end{array}$ & $\mathrm{O}_{2}(\mathrm{mg} / \mathrm{L})$ & $\begin{array}{c}\text { Conductividad } \\
(\mu \mathrm{S} / \mathrm{cm})\end{array}$ & $\operatorname{TDS}(\mathrm{g} / \mathrm{L})$ & $\mathbf{p H}$ & Riqueza \\
\hline AV & $24,4 \pm 1,2 \mathrm{a}$ & $7,9 \pm 0,3 \mathrm{a}$ & $21,1 \pm 3,5 \mathrm{a}$ & $13,9 \pm 1,9 \mathrm{a}$ & $6,4 \pm 0,3 \mathrm{a}$ & $11 \pm 1 \mathrm{a}$ \\
\hline V & $29,9 \pm 0,6 b$ & $6,6 \pm 0,1 b$ & $653,6 \pm 23,2 b$ & $401,6 \pm 4,9 b$ & $7,8 \pm 0,8 b$ & $7 \pm 1 b$ \\
\hline DV & $25,2 \pm 1,1 \mathrm{a}$ & $7,8 \pm 0,1 \mathrm{a}$ & $74,1 \pm 11,2 \mathrm{c}$ & $30,3 \pm 18,9 c$ & $8,2 \pm 0,8 b$ & $9 \pm 1 c$ \\
\hline $\begin{array}{l}\text { ANOV } \\
\text { entre sitios }\end{array}$ & $\mathrm{P}<0.001 * *$ & $\mathrm{P}<0.001^{* *}$ & $\mathrm{P}<0.001^{* *}$ & $\mathrm{P}<0.001^{* *}$ & $\mathrm{P}<0.01^{*}$ & $\mathrm{P}<0.001^{* * *}$ \\
\hline
\end{tabular}

Antes del vertimiento $(\mathrm{AV})$, vertimiento $(\mathrm{V})$ y después del vertimiento (DV)

** Diferencias altamente significativas $(P<0.001)$. * Diferencias significativas $(P<0.05)$

a,b,c Diferentes letras entre columnas indican diferencia significativa según prueba de Tukey $(P<0.05)$

Tabla 2. Resumen estadístico de los ejes para el Análisis de Correspondencia Canónica (CCA) de la abundancia de algas del río Acacias durante época de lluvias

\begin{tabular}{|c|c|c|c|c|c|}
\hline \multirow[b]{2}{*}{ TAXÓN } & \multirow[b]{2}{*}{ ABREVIACIÓN } & \multirow[b]{2}{*}{ CLASE } & \multicolumn{3}{|c|}{ SITIO DE MUESTREO } \\
\hline & & & AV & V & DV \\
\hline Frustula & Frust_sp1 & Bacillariophyceae & $\mathrm{x}$ & $\mathrm{x}$ & $x$ \\
\hline Navicula sp1 & Navi_sp1 & Bacillariophyceae & $x$ & & \\
\hline Navicula sp 2 & Navi_sp2 & Bacillariophyceae & $x$ & & $x$ \\
\hline Navicula sp 3 & Navi_sp3 & Bacillariophyceae & $\mathrm{x}$ & $x$ & \\
\hline Navicula sp 4 & Navi_sp4 & Bacillariophyceae & & & $\mathrm{x}$ \\
\hline Morfoespecie 1 & Bacilla_sp1 & Bacillariophyceae & & & $\mathrm{x}$ \\
\hline Morfoespecie 2 & Bacilla sp2 & Bacillariophyceae & $x$ & & \\
\hline Morfoespecie 3 & Bacilla_sp3 & Bacillariophyceae & $x$ & & $x$ \\
\hline Morfoespecie 4 & Bacilla_sp4 & Bacillariophyceae & $x$ & $x$ & $x$ \\
\hline Desmodesmus sp & Desmo_sp1 & Chorophyceae & $x$ & & $x$ \\
\hline Cosmariumsp & Cosma_sp1 & Zygnemathophyceae & $x$ & & \\
\hline Oedog onium sp1 & Oedog_sp1 & Oedogoniophyceae & $x$ & $x$ & $\mathrm{x}$ \\
\hline Oedog onium sp2 & Oedog_sp2 & Oedogoniophyceae & $x$ & $x$ & $\mathrm{x}$ \\
\hline Borzia sp1 & Borzia_sp1 & Cyanophycecae & & $x$ & \\
\hline Borzia sp2 & Borzia_sp2 & Cyanophycecae & & $x$ & \\
\hline Nostoc sp & Nostoc_sp1 & Cyanophycecae & & $x$ & \\
\hline
\end{tabular}

Antes del vertimiento (AV), en el vertimiento $(\mathrm{V})$ y después del vertimiento (DV) 
La abundancia relativa no varió de manera significativa en cada uno de los grupos algales en los tres lugares evaluados durante los cinco muestreos realizados (Figura 2).

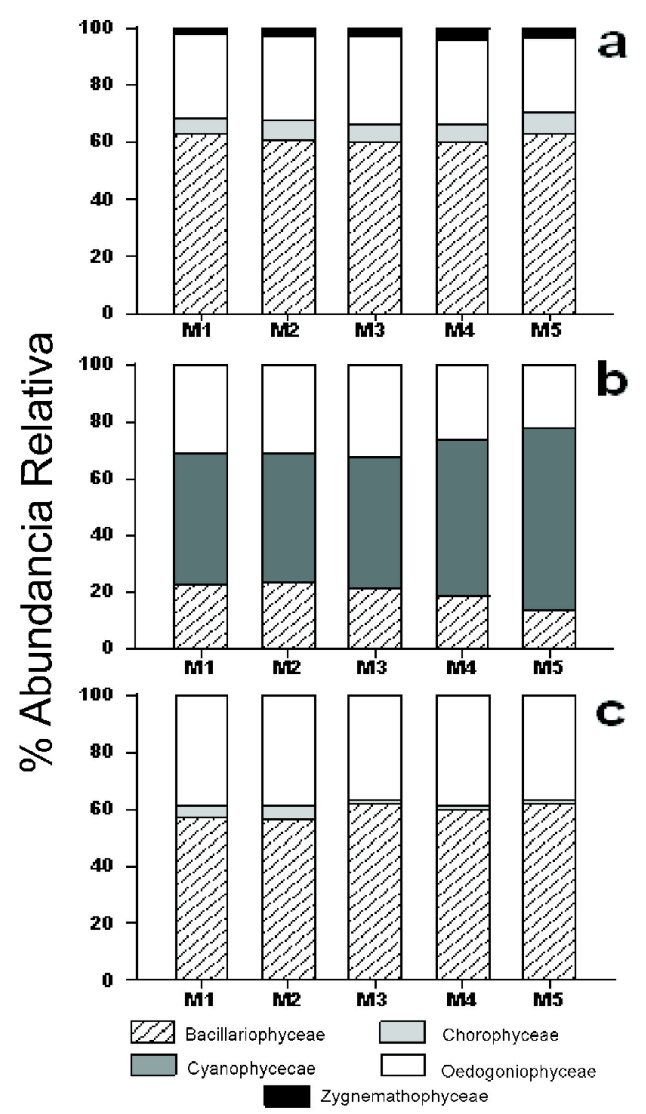

Figura 2. Porcentaje promedio de las abundancias para cada uno de los 5 días de muestreo (M1, M2, M3, M4 y M5) en los tres puntos evaluados del río Acacias durante época de lluvias: (a) antes del vertimiento, (b) en el vertimiento y (c) después del vertimiento

\section{Variables Físicas y Químicas}

La temperatura, la conductividad y los sólidos totales disueltos presentaron valores significativamente superiores en el vertimiento, contrario a lo observado en los puntos antes del vertimiento y después del vertimiento $(P<0.001)$; sin embargo, el oxígeno disuelto exhibió un comportamiento opuesto, presentando valores significativamente más bajos en el vertimiento $(P<0.001)$, cuando comparado con los otros dos puntos de muestreo (Tabla 1).
Los lugares antes y después del vertimiento presentaron los valores más bajos de conductividad, sólidos totales disueltos y temperatura, así como los valores más elevados de riqueza (Figura 3A, 3B y $3 \mathrm{C}$ ). En estos tramos también se presentaron los valores de oxigeno disuelto más elevados (Figura 3D). 

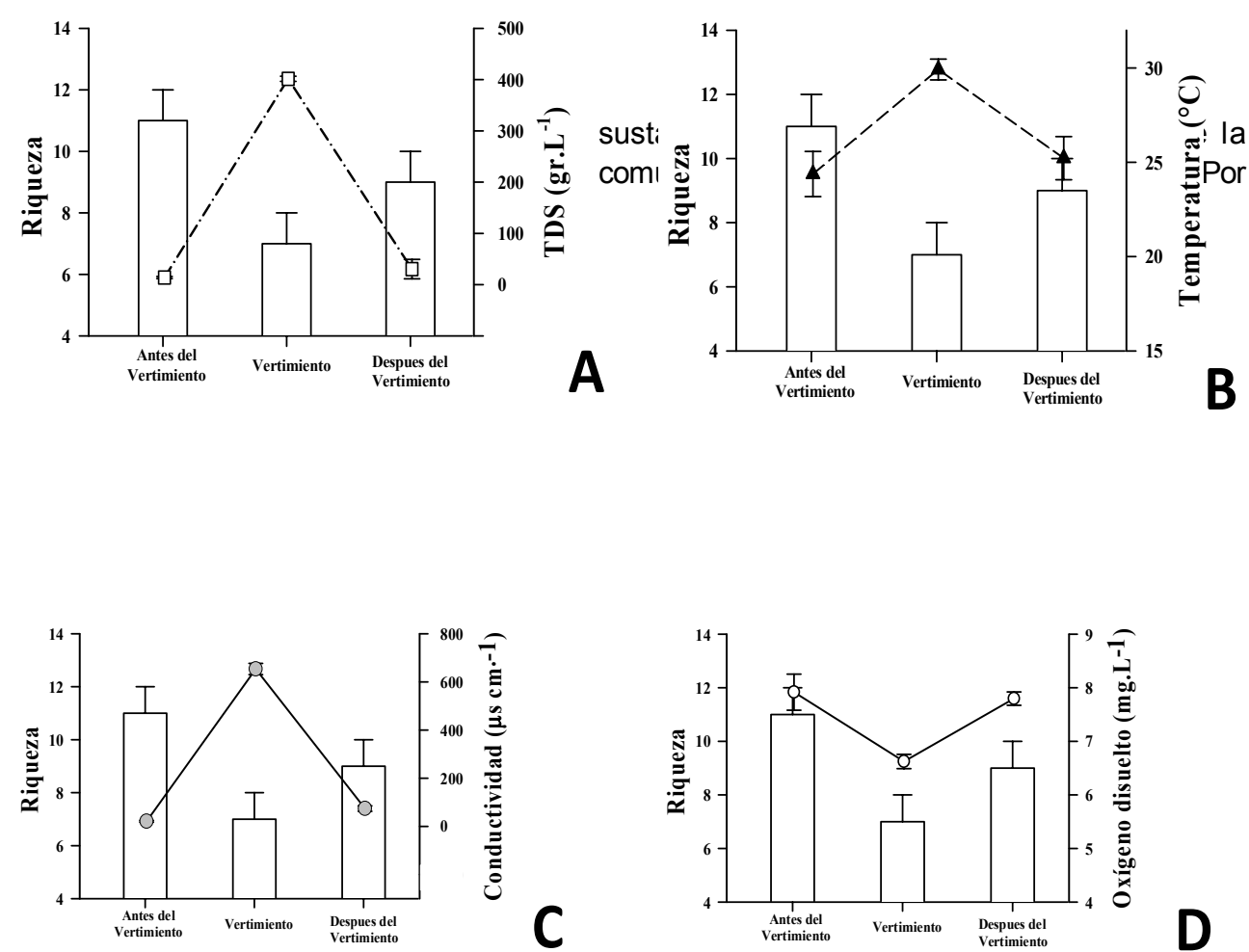

D

Figura 3. Comparación de la riqueza (mostrada en barras) y las variables (mostrada en puntos) de A, sólidos totales disueltos (TSD); $\mathrm{B}$, temperatura; $\mathrm{C}$, conductividad y $\mathrm{D}$, oxígeno disuelto en cada uno de los puntos de muestreo del río Acacias durante época de lluvias

\section{Análisis de Correspondencia Canónica}

El resumen estadístico del CCA se muestra en la Tabla 3. Los ejes 1, 2 y 3 evaluados con el test de Monte Carlo son altamente significativos $(P<0.01)$. La varianza total explicada por la frecuencia especie-ambiente para los dos primeros ejes fue $99.1 \%$. En el primer eje se encontró una fuerte correlación positiva con la conductividad, la temperatura y los sólidos totales disueltos y una correlación negativa con el oxígeno disuelto. El segundo eje presentó una fuerte correlación negativa con el $\mathrm{pH}$.

El triplot basado en el ACC de las variables físicas y químicas y especies fitoperifíticas más representativas se ilustra en la Figura 4 . En la correlación de las variables físicas y químicas y la distribución de las especies se observa un primer grupo (lado derecho del plot) formado por las Cyanophycecae presentes únicamente en el vertimiento cuya presencia puede correlacionarse con la alta conductividad, la temperatura y los sólidos totales disueltos. Por otra parte, se observan los organismos ubicados en los lugares antes y después del vertimiento (lado izquierdo del plot), correlacionados con la concentración de oxígeno disuelto y el $\mathrm{pH}$, respectivamente. En el centro de la figura se observa la pequeña agrupación de los organismos Oedogonium sp., que fueron comunes en los tres lugares evaluados y que no presentaron una correlación con ninguna de las variables físicas y químicas estudiadas. 
Tabla 3. Resumen estadístico de los ejes para el Análisis de Correspondencia Canónica (CCA) de la abundancia de algas del río Acacias durante época de lluvias

\begin{tabular}{|c|c|c|c|}
\hline & Eje 1 & Eje 2 & Eje 3 \\
\hline Eigenvalue & 0,49 & 0,15 & 0,01 \\
\hline Correlación de Pearson especies-ambiente & 0,99 & 0,85 & 0,76 \\
\hline \multicolumn{4}{|l|}{ Varianza de datos de especies } \\
\hline$\%$ explicada acumulada & 68,2 & 88,1 & 88,6 \\
\hline \multicolumn{4}{|l|}{ Varianza de datos de especies-ambiente } \\
\hline$\%$ explicada acumulada & 76,7 & 99,1 & 99,6 \\
\hline \multicolumn{4}{|l|}{ Correlación intra grupos para variables ambientales } \\
\hline Oxígeno Disuelto & -0.9474 & 0.1206 & -0.2918 \\
\hline Conductividad & 0.9987 & -0.0380 & -0.0237 \\
\hline $\mathrm{pH}$ & 0.2044 & -0.8357 & -0.2156 \\
\hline Temperatura $\left({ }^{\circ} \mathrm{C}\right)$ & 0.9062 & -0.0763 & -0.1819 \\
\hline Sólidos totales disueltos (TDS) & 0.9942 & 0.0021 & -0.0193 \\
\hline
\end{tabular}

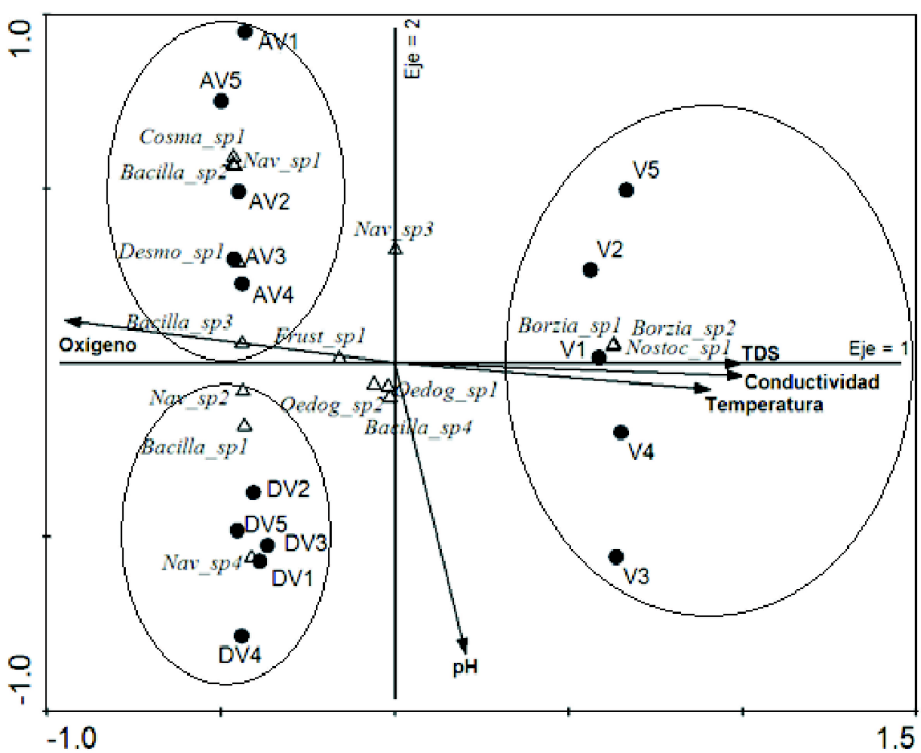

Figura 4. Triplot basado en el Análisis de Correspondencia Canónica (ACC) de las variables físicas y químicas y especies fitoperifíticas más representativas del río Acacias durante época de lluvias. Antes del vertimiento (AV), en el vertimiento (V) y después del vertimiento (DV). El grupo de la derecha ilustra la presencia de Cyanophycecae únicamente en el vertimiento 


\section{DISCUSIÓN}

En general, los bajos valores de riqueza y de abundancia encontrados en el lugar del vertimiento corroboran los posibles efectos deletéreos de los vertimientos sobre el ecosistema acuático analizado, modificando las variables físicas y químicas del ecosistema y por ende de la comunidad algal, la cual respondió a estos cambios. Resultados similares fueron reportados por Naundorf (2002), quien observó que la abundancia y diversidad de microalgas variaron drásticamente en aguas contaminadas.

Los valores intermedios observados tanto en las variables físicas y químicas, como en los factores abióticos en el lugar después del vertimiento, permiten inferir una rápida recuperación del ecosistema acuático, la cual puede ser facilitada por efecto de la mezcla y dilución del agua de producción con el caudal propio del río, permitiendo la recuperación de los valores "normales" de las variables físicas y químicas del agua del afluente. Lavoie et al. (2005) plantearon que la principal fuente de variación en la composición de las Bacillariophyceae, la constituye las diferencias en las condiciones químicas y físicas del hábitat acuático, esto es fácilmente observable en los resultados del presente trabajo, al observarse que los valores más bajos de riqueza y abundancia para este grupo algal se presentaron en el lugar del vertimiento, siendo este sitio el que mayor variación presentó en los parámetros físicos y químicos con respecto a los otros dos sitios analizados.

El género Cosmarium presentó una alta sensibilidad a los efectos del vertimiento, debido a que este grupo sólo fue encontrado en el lugar antes del vertimiento, por lo cual podría ser usado como bioindicador en la evaluación de vertimientos de las industrias petroleras. Sin embargo, la presencia

\section{AGRADECIMIENTOS}

Los autores agradecen a la Empresa Colombiana de Petróleos (ECOPETROL), al Instituto Colombiano del Petróleo (ICP) y al Instituto de Investigaciones de la Orinoquia Colombiana (IIOC) por el aporto económico y al Instituto de Acuicultura del género Oedogonium no varió entre los diferentes lugares, los cual puede significar que este organismo puede tolerar más efectivamente estos vertimientos y por lo tanto tendría un bajo potencial bioindicador de efluentes de industrias petroleras. La conductividad, la temperatura y los sólidos totales disueltos presentaron un comportamiento inversamente proporcional con la riqueza fitoperifitica en cada uno de los sitios muestreados; por el contrario, la concentración de oxígeno disuelto presentó un comportamiento directamente proporcional con este factor biótico.

En conclusión, el agua asociada a la producción petrolera, vertida al río Acacias, está afectando la composición de las comunidades fitoperifíticas de este afluente, lo cual se evidencia por la ausencia de especies que potencialmente podrían desarrollarse en el lugar del vertimiento. Puesto que no se evaluó la composición de las aguas de producción vertidas al afluente, aún no es posible establecer cuál o cuáles serían los agentes o sustancias causantes de este efecto sobre la comunidad fitoperifitica. Sin embargo, se puede inferir que estas aguas de producción contienen altos valores de nitrógeno y fósforo, lo cual provocarían la eutrofización del sistema y podrían estimular el afloramiento de algas Cyanophyceae como lo observado en el lugar del vertimiento, siendo este grupo algal usado como bioindicador de la eutroficación de aguas (Pinilla, 1998).

Por último, cabe proponer para estudios posteriores el análisis de la composición química de las aguas de producción y la realización de muestreos similares durante la época seca del año en esta región (diciembre - marzo), con el fin de proporcionar una visión más completa de los factores influyentes en la comunidad fitoperifítica.

de la Universidad de los Llanos, por el apoyo logístico para la realización de este trabajo. Este trabajo se realizó en el marco del Convenio No. 0008 de 2008, suscrito entre ECOPETROL - ICP UNILLANOS, para la ejecución del proyecto de 
investigación titulado: "Evaluación de una especie fitoplactonica nativa de la Orinoquia colombiana

\section{REFERENCIAS}

Barbour MT, Gerritsen J, Snyder BD, Stribling JB. 1999. Rapid Bioassessment protocols for use in streams and wadeable rivers: Periphyton, Benthic Macroinvertebrates and Fish. Second Edition. EPA 841-B-99-002. U.S. Environmental Protection Agency; Office of Water, Washington, D.C.

French-McCay DP. Development and application of an oil toxicity and exposure model, OILTOXEX. Environ. Toxicol. Chem. 2002; 21: 2080-2094.

Lavoie I, Somers KM, Paterson AM, Dillon PJ. Assessing scales of variability in benthic diatom community structure. J. Appl. Phycol. 2005; 17: 509513.

Lowe R. 1996. Periphyton Patterns in Lakes. In: Algal ecology: Freshwater Bentic Ecosystems. An Introduction to Algal Ecology in Freswater Benthic Habitats. Stevenson et al (eds). Academic Press, inc. California. 57-76 pp.

Naundorf G. 2002. Las comunidades microbianas, fitoplanctónicas y perifíticas en ecosistemas acuáticos. Universidad del Cauca. Material del Curso Biota Acuática I. Popayán, Colombia: Maestría en Recursos Hídricos Continentales. como bioindicador de la toxicidad en los cuerpos de aguas de uso potencial para la acuicultura"

Oliveira-Filho EC, Da-MattaAC, Cabral LL, Veiga LF, Paumgartten FJR. Comparison between four and seven-day Ceriodaphnia dubia survival and reproduction test protocols using oil refinery effluent samples. Braz. Arch. Biol. Techn. 2008; 51: 137-142.

Pinilla G. 1998. Indicadores biológicos en ecosistemas acuáticos continentales de Colombia. Bogotá, Colombia. Fundación Universidad de Bogotá Jorge Tadeo Lozano, Centro de Investigaciones. $67 \mathrm{pp}$.

Pinilla G. 2000. Indicadores biológicos en ecosistemas acuáticos continentales de Colombia. Fundación Universidad de Bogotá Jorge Tadeo Lozano, Bogotá. 67 pp.

Roldan G. 1992. Fundamentos de limnología neotropical. Editorial Universidad deAntioquia. 529 pp.

Ter Braak CJ, Smilauer P. 1998. CANOCO Version 4.0, Software for Canonical Community Ordination. Microcomputer Power Ithaca. New York.

Wetzel, RG. 2001. Limnology. Tercera edición. Academic Press, New York, USA.

Wetzel RG, Likens GE. 2001. Limnologival Analyses. 3 ed. New York. Springer-Verlag. 